Proceedings of the

International Geometry Center

Vol. 11, no. 1 (2018) pp. 1-11

\title{
A generalized Palais-Smale condition in the Fréchet space setting
}

\author{
Kaveh Eftekharinasab
}

\begin{abstract}
The Palais-Smale condition was introduced by Palais and Smale in the mid-sixties and applied to an extension of Morse theory to infinite dimensional Hilbert spaces. Later this condition was extended by Palais for the more general case of real functions over Banach-Finsler manifolds in order to obtain Lusternik-Schnirelman theory in this setting. Despite the importance of Fréchet spaces, critical point theories have not been developed yet in these spaces.

Our aim in this paper is to extend the Palais-Smale condition to the cases of $C^{1}$-functionals on Fréchet spaces and Fréchet-Finsler manifolds of class $C^{1}$. The difficulty in the Fréchet setting is the lack of a general solvability theory for differential equations. This restricts us to adapt the deformation results (which are essential tools to locate critical points) as they appear as solutions of Cauchy problems. However, Ekeland proved the result, today is known as Ekleand's variational principle, concerning the existence of almostminimums for a wide class of real functions on complete metric spaces. This principle can be used to obtain minimizing Palais-Smale sequences. We use this principle along with the introduced conditions to obtain some customary results concerning the existence of minima in the Fréchet setting.

Recently it has been developed the projective limit techniques to overcome problems (such as solvability theory for differential equations) with Fréchet spaces. The idea of this approach is to represent a Fréchet space as the projective limit of Banach spaces. This approach provides solutions for a wide class of differential equations in every Fréchet space and therefore can be used to obtain deformation results. This method would be the proper framework for further development of critical point theory in the Fréchet setting.
\end{abstract}

Keywords: The Palais-Smale condition, Fréchet-Finsler manifold, Ekeland's variational principle 


\begin{abstract}
Анотація. Умова Паліса-Смейла була введена Р. Палісом та С. Смейлом в середині 60-х та застосована для перенесення теорії Морса на нескінченовимірні гільбертові простори. Пізніше, Р. Паліс узагальнив їі для дійснозначних функцій на многовидах Банаха-Фінслера для того, щоб розширити теорію Люстерніка-Шнірельмана на цей випадок. Незважаючи на важливість просторів Фреше, на даний момент теорія критичних точок для цих просторів розвинена дуже слабо.

Мета даної роботи розширити умову Паліса-Смейла на випадок $C^{1}$ функцій на просторах Фреше та на многовидах Фреше-Фінслера класу $C^{1}$. Складість роботи з просторами Фреше полягає у відсутності для них загальної теорії розв'язності диференціальних рівнянь. Це суттєво обмежує можливості адаптувати для них деформаційні результати (які є суттєвим інструментом для знаходження критичних точок), через те, що вони отримуються як розв'язки проблем Коші.

I. Екеланд довів так званий «варіаційний принцип Екленда», який гарантує існування майже мінімумів для широкого класу дійснозначних функцій на повних метричних просторах. Цей принцип може бути використаний для отримання мінімізуючих послідовностей ПалісаСмейла. В даній роботі ми використовуємо цей принцип разом 3 додатковою умовою для того, щоб отримати деякі результати про існування мінімумів функцій на просторах Фреше.

Розвинена в останні роки техніка проективних границь дозволяє обійти проблеми роз'язності диференціальних рівнянь в просторах Фреше. Ідея полягає в тому, щоб представити простір Фреше як проективну границю банахових просторів. Такий підхід дозволяє розв'язати широкий клас диференціальних рівнянь в кожному просторі Фреше, а отже, може використовуватись для отримання деформаційних результатів. Описаний метод має потенціальні застосування для розвитку теорії критичних точок в просторах Фреше.
\end{abstract}

\title{
1. INTRODUCTION
}

The Palais-Smale condition is an essential tool for critical point theory in infinite dimensional spaces. Since it was established by Palais and Smale [8], a number of its generalizations for various categories of maps have been introduced (cf. [6] for a survey). The Palais-Smale type conditions have been applied to numerous problems in critical points theory and its applications to various problems in differential equations, geometry and physics (see [6]).

In this paper we generalize the Palais-Smale condition to the cases of $C^{1}$-functionals on Fréchet spaces and Fréchet-Finsler manifolds of class $C^{1}$. It is instantly noticed that due to the weak structural constraints of Fréchet spaces, applications of the conditions are too restrictive. It is well known that some of the basic tools for locating critical points of $C^{1}$-functionals are the deformation lemmas. The deformations that appear in the deformation lemmas are obtained as solutions of Cauchy problems. However, 
on Fréchet spaces, an ordinary differential equation may admit no, one or multiple solutions for the same initial condition. Thus, we can not get deformation results for Fréchet spaces in general. However, we can still use the introduced conditions along with Ekeland's variational principle to locate critical points. We then apply them and the works of Ekeland [2] and Qui [9] to state some standard results dealing with the existence of minima.

\section{Prerequisites}

We use the following notion of $C^{k}$-maps in Michal-Bastiani sense. We shall work in the category of manifolds of class at least $C^{1}$.

Definition 2.1. Let $E$ and $F$ be Fréchet spaces, $U \subseteq E$ be an open subset and $f: U \rightarrow F$ be a continuous map. The derivative of $f$ at $x \in U$ in the direction of $h \in E$ is defined as

$$
\mathrm{d} f(x)(h):=\lim _{t \rightarrow 0} \frac{1}{t}(f(x+h t)-f(x)),
$$

whenever the limit exits. The map $f$ is called differentiable at $x$ if $\mathrm{d} f(x)(h)$ exists for all $h \in E$. It is called a $C^{1}-m a p$ if it is differentiable at all points of $U$ and

$$
\mathrm{d} f: U \times E \rightarrow F, \quad(x, h) \mapsto \mathrm{d} f(x)(h),
$$

is a continuous map. The map $f$ is called a $C^{k}-m a p, k \geq 2$, if it is continuous, the higher directional derivatives

$$
\mathrm{d}^{k} f(x)\left(h_{1}, \ldots, h_{k}\right):=\lim _{t \rightarrow 0} \frac{\mathrm{d}^{k-1} f\left(x+h_{k} t\right)\left(h_{1}, \ldots, h_{k-1}\right)-\mathrm{d}^{k-1} f(x)\left(h_{1}, \ldots, h_{k-1}\right)}{t}
$$

exist for all integers $j \leqq k, x \in U$ and $h_{1}, \ldots, h_{j} \in E$, and all maps

$$
\mathrm{d}^{j} f: U \times E^{j} \rightarrow F, \quad\left(x, h_{1}, \ldots, h_{j}\right) \mapsto \mathrm{d}^{j} f(x)\left(h_{1}, \ldots, h_{j}\right),
$$

are continuous. We say that $f$ is smooth or $C^{\infty}$ if it is $C^{k}$ for all $k \in \mathbb{N}$.

Within this framework most of the notions of the theory of infinite dimensional manifolds are sufficiently well behaved for our purposes. In particular, Fréchet manifolds, Fréchet tangent bundles and $C^{k}$-maps between Fréchet manifolds are defined in obvious way (cf. [3]). However, for a Fréchet manifold $M$ modelled on a Fréchet space $F$ we can define the set-theoretic cotangent bundle

$$
T^{\prime} M:=\bigcup_{m \in M}\left(T_{m} M\right)^{\prime},
$$

where $\left(T_{m} M\right)^{\prime}$ is the dual of a tangent space $T_{m} M$. As a set, it carries a natural structure of a vector bundle over $M$, but in general there is no vector topology on $F^{\prime}$, the dual of $F$, that can lead to the identification $T^{\prime} M \cong$ $F \times F^{\prime}$. Therefore there is no natural smooth vector bundle structure on 
$T^{\prime} M$ if $F$ is not normable, see [7, Remark I.3.9]. Thus instead, we use the notion of a $\mathcal{B}$-cotangent bundle [10]. In this definition to put a manifold structure on $T^{\prime} M$, the dual of $F$ is equipped by a $\mathcal{B}$-topology, where $\mathcal{B}$ is a bornology on $F$. To be precise, we recall that a family $\mathcal{B}$ of subsets of $F$ is called a bornology on $F$ if the followings conditions are satisfied

(1) $\bigcup_{B \in \mathcal{B}}=F$,

(2) for every $A, B \in \mathcal{B}$ there exists $C \in \mathcal{B}$ such that $A \cup B \subset C$,

(3) for all $B \in \mathcal{B}$ and $r \in \mathbb{R}$ there exists $C \in \mathcal{B}$ such that $r \cdot B \subset C$.

Let $E$ be a Fréchet space, $\mathcal{B}$ be a bornology on $E$ and $L_{\mathcal{B}}(E, F)$ be the space of all linear continuous maps from $E$ to $F$. The $\mathcal{B}$-topology on $L_{\mathcal{B}}(E, F)$ is a Hausdorff locally convex topology defined by all seminorms obtained as follows:

$$
\mathrm{P}_{B}^{n}(L):=\sup \left\{\mathrm{p}_{n}(L(e)) \mid e \in B\right\},
$$

where $B \in \mathcal{B}$ and $\left\{\mathrm{p}_{n}\right\}_{n \in \mathbb{N}}$ is a family of seminorms defining the topology of $F$. One similarly may define $L_{\mathcal{B}}^{k}(E, F)$ and $\bigwedge^{k} L_{\mathcal{B}}(E, \mathbb{R})$, the space of $k$ linear jointly continuous maps of $E^{k}$ to $F$ and the space of anti-symmetric $k$-linear jointly continuous maps of $E^{k}$ to $\mathbb{R}$, respectively. If $\mathcal{B}$ contains all compact sets, then the $\mathcal{B}$-topology on the space $L_{\mathcal{B}}(E, \mathbb{R})=E_{\mathcal{B}}^{\prime}$ of all continuous linear functional on $E$, the dual of $E$, is the topology of compact convergence. For this and further details on bornologies we refer to [4].

If $\mathcal{B}$ contains all compact sets of $E$, then $L_{\mathcal{B}}^{k}\left(E, L_{\mathcal{B}}^{l}(E, F)\right)$ is canonically isomorphic to $L_{\mathcal{B}}^{l+k}(E, F)$ as a topological vector space, see [5, Theorem 0.1.3]. In particular, $L_{\mathcal{B}}^{2}(E, \mathbb{R})=L_{\mathcal{B}}^{2}(E) \cong L_{\mathcal{B}}\left(E, E_{\mathcal{B}}^{\prime}\right)$. Under the above condition on $\mathcal{B}$, we define the differentiability of class $C_{\mathcal{B}}^{k}$ as follows.

Let $U \subset E$ be an open set. Then a map $f: U \rightarrow F$ is called $C_{\mathcal{B}}^{1}$ if its partial derivatives exist and the induced map $\mathrm{d} f: U \rightarrow L_{\mathcal{B}}(E, F)$ is continuous. Similarly we can define maps of class $C_{\mathcal{B}}^{k}, k \in \mathbb{N} \cup\{\infty\}$, see $[5$, Definition 2.5.0]. A map $f: U \rightarrow F$ is $C_{\mathcal{B}}^{k}, k \geqq 1$, if and only if $f$ is $C^{k}$ in the sense of Definition (2.1), see [5, Theorem 2.7.0 and Corollary 1.0.4 (2)]. In particular, $f$ is $C_{\mathcal{B}}^{\infty}$ if and only in $f$ is $C^{\infty}$. Thus, if $f$ at $x \in E$ is $C^{1}$ and hence $C_{\mathcal{B}}^{1}$, the derivative $f$ at $x, \mathrm{~d} f(x)$, is an element of $E_{\mathcal{B}}^{\prime}$, and we shall denote $\mathrm{d} f(x)$ evaluated at $h \in E$ via $\mathrm{d} f(x)(h)$.

Assume that $\mathcal{B}$ is a bornology on $F$ containing all compact sets and $M$ is a Fréchet manifold modelled on $F$. Let $f$ be a functional defined over $M$. The derivative of $f$ at $x \in M$ can be written in terms of the iterated tangent bundles of $M$ and we can consider $\mathrm{d} f: T M \rightarrow \mathbb{R}$ given by $\mathrm{d} f(x, h)=\mathrm{d} f(x)(h)$ upon locally identifying $T M$ with $U \times F$, where $U$ is an open set in $F$. Therefore, if at $x \in M$ a map $f: M \rightarrow \mathbb{R}$ is $C^{1}$ and hence $C_{\mathcal{B}}^{1}$, then $\mathrm{d} f(x)$ belongs to $L_{\mathcal{B}}\left(T_{x} M, \mathbb{R}\right)=\left(T_{x} M\right)_{\mathcal{B}}^{\prime}$. 
Definition 2.2. Let $M$ be a Fréchet manifold modelled on a Fréchet space $F$ and $\mathcal{B}$ a bornology on $F$. The $\mathcal{B}$-cotangent bundle of $M$ is defined as $T M_{\mathcal{B}}^{\prime}:=\bigcup_{x \in M}\left(T_{x} M\right)_{\mathcal{B}}^{\prime}$ and the $k$-exterior product of the $\mathcal{B}$-cotangent bundle as $\bigwedge^{k} T M_{\mathcal{B}}^{\prime}:=\bigcup_{x \in M} \bigwedge^{k}\left(T_{x} M\right)_{\mathcal{B}}^{\prime}$.

If $\mathcal{B}$ is chosen such that $T(\mathcal{B}) \subset \mathcal{B}$ for all continuous linear endomorphisms $T$ of $F$, then $\bigwedge^{k} T M_{\mathcal{B}}^{\prime}$ is a vector bundle in the category of locally convex spaces with the local model $F \times \bigwedge^{k} F_{\mathcal{B}}^{\prime}$. In particular, $T M_{\mathcal{B}}^{\prime}$ is a vector bundle in the category of locally convex spaces with the local model $F \times F_{\mathcal{B}}^{\prime}$, see [10, Remark (1), p. 60].

\section{The Palais-Smale Condition}

A Finsler structure for a Fréchet manifold $M$ in the sense of Palais was defined in [1]. We now define a Finsler structure on its $\mathcal{B}$-cotangent bundle.

Henceforth, we always assume that a bornology $\mathcal{B}$ on a Fréchet space $F$ contains all compact sets and we always chose $\mathcal{B}$ such that $T(\mathcal{B}) \subset \mathcal{B}$ for all continuous linear endomorphisms $T$ of $F$ whenever we define the $\mathcal{B}$-cotangent bundle of a manifold $M$ modelled on $F$.

Definition 3.1. Let $M$ be a $C^{k}$ Fréchet manifold modelled on a Fréchet space $F$, where $k \in \mathbb{N} \cup\{\infty\}$. A Finsler structure for $M$ is a collection of functions $\|\cdot\|_{M}^{n}: T M \rightarrow \mathbb{R}^{+}, n \in \mathbb{N}$, on its tangent bundle $T M$ such that

(1) for each $x \in M$ and $n \in \mathbb{N}$, the function

$$
\|\cdot\|_{x}^{n}:=\|\cdot\|_{\left.M\right|_{T_{x} M}}^{n}: T_{x} M \rightarrow \mathbb{R}^{+}
$$

is a seminorm on the tangent space $T_{x} M$ such that for every chart $\varphi: U \rightarrow F$ with $x \in U$, the collection of seminorms

$$
v \in F \mapsto\left\|\mathrm{d} \varphi^{-1}(\varphi(x))(v)\right\|_{x}
$$

generates the topology of $F$;

(2) given $K>1, x_{0} \in M$ and a chart $\varphi: U \rightarrow F$ with $x_{0} \in U$, there exists an open neighborhood $\mathcal{U}$ of $x_{0}$ in $U$ such that

$$
\frac{1}{K}\left\|\mathrm{~d} \varphi^{-1}\left(\varphi\left(x_{0}\right)\right)(v)\right\|_{x_{0}}^{n} \leqq\left\|\mathrm{~d} \varphi^{-1}(\varphi(x))(v)\right\|_{x}^{n} \leqq K\left\|\mathrm{~d} \varphi^{-1}\left(\varphi\left(x_{0}\right)\right)(v)\right\|_{x_{0}}^{n}
$$

for all $x \in \mathcal{U}, n \in \mathbb{N}, v \in F$.

If $\left\{\|\cdot\|^{n}\right\}_{n \in \mathbb{N}}$ is a Finsler structure on $T M$, then eventually we can obtain a graded Finsler structure, denoted by $\left(\|\cdot\|^{n}\right)_{n \in \mathbb{N}}$, on $T M$. A Fréchet manifold $M$ is called Fréchet-Finsler if its tangent bundle $T M$ admits a Finsler structure. If a Fréchet manifold $M$ is paracompact and its model space $F$ is such that all seminorms defining the topology of $F$ are smooth maps on $F \backslash\{0\}$, then $T M$ admits a Finsler structure ([1, Proposition 
4]). As an example of a $C^{k}$-Finsler manifold we can consider any $C^{k}$ submanifold $M$ of a Fréchet space $F$, with $T_{m} M$ carrying the collection of seminorms induced by the inclusion $T_{m} M \subset T_{m} F \cong F$.

Let $M$ be a connected Fréchet-Finsler manifold endowed with a graded Finsler structure $\left(\|\cdot\|^{n}\right)_{n \in \mathbb{N}}$. For each $n \in \mathbb{N}$ define $d^{n}(x, y)=\inf _{\gamma} L^{n}(\gamma)$, where the infimum is taken over all $C^{1}$-curves $\gamma:[a, b] \rightarrow M$ such that $\gamma(a)=x, \gamma(b)=y$ and

$$
L^{n}(\gamma)=\int_{a}^{b}\left\|\left(\gamma^{\prime}(t)\right)\right\|_{\gamma(t)}^{n} \mathrm{~d} t
$$

Therefore, we obtain an increasing sequence of pseudometric $\left(d^{n}(x, y)\right)_{n \in \mathbb{N}}$. Define

$$
\rho(x, y)=\sum_{n=1}^{n=\infty} \frac{1}{2^{n}} \frac{d^{n}(x, y)}{1+d^{n}(x, y)} .
$$

This is a metric, called the Finsler metric, consistent with the original topology of $M([1])$. In the sequel, we say that $M$ is complete if $M$ is complete with respect to this metric. We say that $\rho$ is locally compatible if for each $p \in M$ there exist a chart $(p \in U, \varphi)$ and constants $\alpha, \beta$ such that for all $n \in \mathbb{N}$ and $x, y \in U$

$$
\alpha \rho(x, y) \leqq \mathrm{p}_{n}(\varphi(x)-\varphi(y)) \leqq \beta \rho(x, y),
$$

where $\left\{\mathrm{p}_{n}\right\}_{n \in \mathbb{N}}$ is a family of seminorms defining the topology of $F$.

The $\mathcal{B}$-cotangent bundle $T M_{\mathcal{B}}^{\prime}$ of $M$ carries a natural Finsler structure, denoted again by $\left\{\|\cdot\|^{n}\right\}_{n \in \mathbb{N}}$, characterized by letting

$$
\|w\|_{x}^{n}=\sup \left\{|\langle w, v\rangle| ; v \in T_{x} M,\|v\|_{x}^{n}=1\right\},
$$

where $x \in M, w \in\left(T_{x} M\right)_{\mathcal{B}}^{\prime}$ and $\langle\cdot, \cdot\rangle$ is the duality pairing between $T_{x} M$ and $\left(T_{x} M\right)_{\mathcal{B}}^{\prime}$ which is separately continuous. Consequently, for a $C^{1}$-functional $f: M \rightarrow \mathbb{R}$ the mappings $\|\mathrm{d} f\|_{x}^{n}: M \rightarrow \mathbb{R}$ given by $x \mapsto\|\mathrm{d} f(x)\|_{x}^{n}$ are well defined and continuous for all $n \in \mathbb{N}$. We shall omit the index $x$ in $\|\mathrm{d} f(x)\|_{x}^{n}$ and denote it just by $\|\mathrm{d} f(x)\|^{n}$.

Definition 3.2. Let $F$ be a Fréchet space, $\mathcal{B}$ a bornology on $F$ and $F_{\mathcal{B}}^{\prime}$ the dual of $F$ equipped with the $\mathcal{B}$-topology. Let $f: F \rightarrow \mathbb{R}$ be a $C^{1}$-functional.

(i) We say that $f$ satisfies the Palais-Smale condition, (PS) in short, if each sequence $\left(x_{i}\right) \subset F$ such that $f\left(x_{i}\right)$ is bounded and

$$
\mathrm{d} f\left(x_{i}\right) \rightarrow 0 \text { in } F_{\mathcal{B}}^{\prime},
$$

has a convergent subsequence.

(ii) We say that $f$ satisfies the Palais-Smale condition at level $c \in \mathbb{R}$, $(\mathrm{PS})_{c}$ in short, if each sequence $\left(x_{i}\right) \subset F$ such that

$$
f\left(x_{i}\right) \rightarrow c \text { and } \mathrm{d} f\left(x_{i}\right) \rightarrow 0 \text { in } F_{\mathcal{B}}^{\prime},
$$


has a convergent subsequence.

The above definition of Palais-Smale condition can easily be extended to the more general setting of $C^{1}$-functionals on manifolds with Finsler structures. Thus we may apply the Palais approach [8] to the critical point theory for the general case of Fréchet manifolds.

Definition 3.3. Let $M$ be a connected Fréchet-Finsler manifold endowed with a Finsler structure $\left\{\|\cdot\|^{n}\right\}_{n \in \mathbb{N}}$ and the corresponding Finsler metric $\rho$. Let $f: M \rightarrow \mathbb{R}$ be a $C^{1}$-functional.

(i) We say that $f$ satisfies the Palais-Smale condition, (PS) in short, if for all $n \in \mathbb{N}$ each sequence $\left(x_{i}\right) \subset M$ such that $f\left(x_{i}\right)$ is bounded and $\left\|\mathrm{d} f\left(x_{i}\right)\right\|^{n} \rightarrow 0$, has a convergent subsequence.

(ii) We say that $f$ satisfies the Palais-Smale condition at level $c \in \mathbb{R}$, (PS) $\mathrm{c}$ in short, if for all $n \in \mathbb{N}$ each sequence $\left(x_{i}\right) \subset M$ such that $f\left(x_{i}\right) \rightarrow c$ and $\left\|\mathrm{d} f\left(x_{i}\right)\right\|^{n} \rightarrow 0$, has a convergent subsequence.

Let $f$ be a $C^{1}$-functional on a Fréchet space (or a Fréchet-Finsler manifold). As usual, a point $p$ in the domain of $f$ is said to be a critical point of $f$ if $\mathrm{d} f(p)=0$, the corresponding value $c=f(p)$ will be called a critical value. We will use the following standard notation:

$$
K_{c}:=\{p \in M \mid f(p)=c, \mathrm{~d} f(p)=0\} .
$$

\section{Existence of Minima}

Consider the following particular version of Ekeland's variational principle (cf. [2]).

Theorem 4.1. Let $(X, \sigma)$ be a complete metric space. Let a functional $f: X \rightarrow(-\infty, \infty]$ be semi-continuous, bounded from below and not identical to $+\infty$. Let $a>1, b>0$ and $x \in X$ be given such that $f(x) \leqq \inf _{X} f+a$. Then there exists $x_{a} \in M$ such that

(1) $f\left(x_{a}\right) \leqq f(x)$,

(2) $\sigma\left(x_{a}, x\right) \leqq \frac{1}{b}$

(3) $f\left(x_{a}\right)<f(x)+a b \sigma\left(x, x_{a}\right), \quad \forall x \neq x_{a} \in M$.

When $X$ is a complete Fréchet-Finsler manifold and $f$ is of class $C^{1}$, we have the following:

Theorem 4.2. Let $M$ be a connected complete Fréchet-Finsler manifold of class $C^{1}$ endowed with a Finsler structure $\left\{\|\cdot\|^{n}\right\}_{n \in \mathbb{N}}$ and the corresponding Finsler metric $\rho$ which is locally compatible. Let a $C^{1}$-functional $f: M \rightarrow \mathbb{R}$ 
be bounded from below. Then, for each $\theta>1$ and each $m \in M$ such that $f(m) \leqq \inf _{M} f+\theta^{2}$, there exist $m_{\theta} \in M$ and a constant $\varepsilon_{\theta}$ such that

(1) $f\left(m_{\theta}\right) \leqq f(m)$,

(2) $\rho\left(m_{\theta}, m\right) \leqq \theta$,

(3) $\left\|\mathrm{d} f\left(m_{\theta}\right)\right\|^{n} \leqq \varepsilon_{\theta}, \quad \forall n \in \mathbb{N}$.

Proof. Given $\theta>1$, apply Theorem 4.1 with $b=\frac{1}{\theta}$ to find $m_{\theta} \in M$ such that (1) and (2) hold and

$$
f(x)>f\left(m_{\theta}\right)-\theta \rho\left(m_{\theta}, x\right), \quad \forall x \neq m_{\theta} \in M .
$$

Since $\rho$ is locally compatible, there exist a chart $\varphi: U \rightarrow F$ with $m_{\theta} \in U$ ( $F$ is the local model of $M$ ) and constants $\alpha, \beta$ such that for all $n \in \mathbb{N}$ and $x, y \in U$

$$
\alpha \rho(x, y) \leqq \mathrm{p}_{n}(\varphi(x)-\varphi(y)) \leqq \beta \rho(x, y),
$$

where $\left\{\mathrm{p}_{n}\right\}_{n \in \mathbb{N}}$ is a family of seminorms defining the topology of $F$. Let $h \in \varphi(U)$ and $t>0$ be such that $\varphi^{-1}\left(\varphi\left(m_{a}\right)+t h\right) \in U$ Then by (4.1)

$$
f\left(\varphi^{-1}\left(\varphi\left(m_{\theta}\right)\right)\right)-f\left(\varphi^{-1}\left(\varphi\left(m_{\theta}\right)+t h\right)\right)<\theta \rho\left(m_{\theta}, \varphi^{-1}\left(\varphi\left(m_{\theta}\right)+t h\right)\right) .
$$

Hence, for all $n \in \mathbb{N}$

$$
\begin{aligned}
& f\left(\varphi^{-1}\left(\varphi\left(m_{\theta}\right)\right)\right)-f\left(\varphi^{-1}\left(\varphi\left(m_{\theta}\right)+t h\right)\right)< \\
&<\frac{\theta}{\alpha} \mathrm{p}_{n}\left(\varphi\left(m_{\theta}\right)-\left(\varphi\left(m_{\theta}\right)+t h\right)\right)=\frac{\theta}{\alpha} \mathrm{p}_{n}(h) t< \\
& \quad<\frac{\theta}{\alpha} \beta \rho\left(m_{\theta}, \varphi^{-1}\left(\varphi\left(m_{\theta}\right)+t h\right)\right) t<\frac{\theta^{2}}{\alpha} \beta t .
\end{aligned}
$$

Define a $C^{1}$-curve $\gamma:\left[0, t_{0}\right] \rightarrow M$ by $\gamma(t)=\varphi^{-1}\left(\varphi\left(m_{\theta}\right)+t h\right)$ such that $\gamma\left(\left[0, t_{0}\right]\right) \subset U$ and let $\varepsilon_{\theta}=\frac{\theta^{2}}{\alpha} \beta$. By dividing both sides in (4.3) by $t$ and letting $t \rightarrow 0$, we obtain

$$
\mathrm{d} f\left(m_{\theta}\right)(h)=\left.\frac{\mathrm{d}}{\mathrm{d} t}(f \circ \gamma)\right|_{t=0} \geq-\varepsilon_{\theta} .
$$

Changing $h$ with $-h$ gives $\mathrm{d} f\left(m_{\theta}\right)(h) \leqq \varepsilon_{\theta}$, thereby for all $n \in \mathbb{N}$

$$
\left\|\mathrm{d} f\left(m_{\theta}\right)\right\|^{n} \leqq \sup _{\mathrm{p}_{i}(h)=1, i \in \mathbb{N}} \mathrm{d} f\left(m_{\theta}\right)(h) \leqq \varepsilon_{\theta} .
$$

Theorem is proved.

Corollary 4.3. Let $M$ and $f$ be as in Theorem 4.2. If $(\mathrm{PS})_{c}$ holds with $c=\inf _{M} f$, then $f$ attains its minimum at a point in $K_{c}$. 
Proof. According to Theorem 4.2, if for each $i \in \mathbb{N}$ we let $\theta^{2}=\frac{1}{i}$, then there exists $x_{i} \in M$ such that

$$
f\left(x_{i}\right) \leqq c+\frac{1}{i}, \quad\left\|\mathrm{~d} f\left(x_{i}\right)\right\|^{n} \leqq \frac{\beta}{i \alpha}, \quad n \in \mathbb{N} .
$$

Therefore, $f\left(x_{i}\right) \rightarrow c$ and $\left\|\mathrm{d} f\left(x_{i}\right)\right\|^{n} \rightarrow 0$ for all $n \in \mathbb{N}$. Since $f$ satisfies $(\mathrm{PS})_{c}$ it follows that there exists a convergent subsequence $x_{i_{j}} \rightarrow x_{0}$. Hence, $f\left(x_{0}\right)=c=\inf _{M} f$ and $\mathrm{d} f\left(x_{0}\right)=0$.

The similar results but with weaker assumptions can be obtained for Fréchet spaces by means of the following Ekeland's variational principle.

Theorem 4.4 ([9], Corollary 2.1). Let $F$ be a Fréchet space with topology generated by an increasing sequence $\mathrm{p}_{1}(\cdot) \leqq \mathrm{p}_{2}(\cdot) \leqq \cdots$ of seminorms. Let a functional $f: F \rightarrow(-\infty, \infty]$ be semi-continuous, bounded from below and not identical to $+\infty$. Let $\eta>0$ and $x_{0} \in F$ be given such that $f\left(x_{0}\right) \leqq \inf _{F} f+\eta$, and let $\left(\lambda_{n}\right)_{n \in \mathbb{N}}$ be a sequence of positive real numbers. Then for any $i \in \mathbb{N}$, there exists $z \in F$ such that

(1) $\lambda_{j} \mathrm{p}_{j}\left(z-x_{0}\right) \leqq f\left(x_{0}\right)-f(z)$ for $j=1, \ldots, i$;

(2) $\mathrm{p}_{j}\left(z-x_{0}\right)<\frac{\eta}{\lambda_{j}}$ for $j=1, \ldots, i$;

(3) for any $x \in F, x \neq z$, there exists $m \in \mathbb{N}$ such that

$$
\lambda_{m} \mathrm{p}_{m}(x-z)+f(x)>f(z),
$$

or equivalently, $\sup _{n} \lambda_{n} \mathrm{p}_{n}(x-z)+f(x)>f(z)$ for any $x \in F, x \neq z$.

Theorem 4.5. Let $F$ be a Fréchet space with topology generated by an increasing sequence $\mathrm{p}_{1}(\cdot) \leqq \mathrm{p}_{2}(\cdot) \leqq \cdots$ of seminorms. Let $\mathcal{B}$ a bornology on $F$ and $\left\{\mathrm{P}_{B}^{n}\right\}_{n \in \mathbb{N}}$ the family of seminorms (defined as in (2.1)) that generates the topology of $F_{\mathcal{B}}^{\prime}$. Let a $C^{1}$-functional $f: F \rightarrow \mathbb{R}$ be bounded from below. Then, for each $\varepsilon>0, i \in \mathbb{N}, x \in F$ such that $f(x) \leqq \inf _{F} f+\varepsilon$, there exists $z \in F$ such that

(1) $\mathrm{p}_{j}\left(z-x_{0}\right) \leqq \frac{f\left(x_{0}\right)-f(z)}{\sqrt{\varepsilon}}$ for $j=1, \cdots, i$;

(2) $\mathrm{p}_{j}\left(z-x_{0}\right)<\sqrt{\varepsilon}$ for $j=1, \cdots, i$;

(3) $\mathrm{P}_{B}^{n}(\mathrm{~d} f(z)) \leqq \sqrt{\varepsilon}, \quad \forall n \in \mathbb{N}, B \in \mathcal{B}$.

Proof. Given $\varepsilon>0$ and $i \in \mathbb{N}$, apply Theorem 4.4 with $\lambda_{j}=\sqrt{\varepsilon}$ for all $j \in \mathbb{N}$ and $\eta=\varepsilon$ to find $m \in \mathbb{N}$ and $z \in F$ such that (1) and (2) hold and

$$
f(x)>f(z)-\sqrt{\varepsilon} \mathrm{p}_{m}(x-z), \quad \forall x \neq z \in F .
$$


By applying (4.4) to $x=z+t h$ with $t>0$ and $h \in F$, we obtain

$$
f(z+t h)-f(z)>-\sqrt{\varepsilon} \mathrm{p}_{m}(h) t .
$$

Dividing both sides by $t$ and letting $t \rightarrow 0$ yields $\mathrm{d} f(z)(h)>-\sqrt{\varepsilon} \mathrm{p}_{m}(h)$ for all $h \in F$. Changing $h$ with $-h$ gives $\mathrm{d} f\left(m_{\theta}\right)(h) \leqq \sqrt{\varepsilon} \mathrm{p}_{m}(h)$ for all $h \in F$, which means for all $n \in \mathbb{N}$ and $B \in \mathcal{B}$ we have $\mathrm{P}_{B}^{n}(\mathrm{~d} f(z)) \leqq \sqrt{\varepsilon}$.

Remark 4.6. To prove the preceding theorem as in the case of FinslerFréchet manifolds we could use Theorem 4.1. However, that imposes restriction on a metric that defines the Fréchet topology. In Theorem 4.4 we use collections of seminorms rather than metrics. For technical reasons it is more convenient to deal with seminorms.

Corollary 4.7. Let $F$ and $f$ be as in Theorem 4.5. If (PS $)_{c}$ holds with $c=\inf _{F} f$, then $f$ attains its minimum at a point in $K_{c}$.

Proof. According to Theorem 4.5, if for each $i \in \mathbb{N}$ we let $\varepsilon=\frac{1}{i}$. Then there exists $x_{i} \in F$ such that

$$
f\left(x_{i}\right) \leqq c+\frac{1}{i}, \quad \mathrm{P}_{B}^{n}\left(\mathrm{~d} f\left(x_{i}\right)\right) \leqq \frac{1}{\sqrt{i}}, \quad \forall n \in \mathbb{N}, B \in \mathbb{N} .
$$

Therefore, $f\left(x_{i}\right) \rightarrow c$ and $\mathrm{d} f\left(x_{i}\right) \rightarrow 0$ in $F_{\mathcal{B}}^{\prime}$. Since $f$ satisfies $(\mathrm{PS})_{c}$ it follows that there exists a convergent subsequence $x_{i_{j}} \rightarrow x_{0}$.

Hence, $f\left(x_{0}\right)=c=\inf _{M} f$ and $\mathrm{d} f\left(x_{0}\right)=0$.

\section{REFERENCES}

[1] C. L. Bejan. Finsler structures on Fréchet bundles. Proc. 3-rd seminar on Finsler spaces, Univ. Braşov 1984, Societatea de ştiinţe Matematice Romania, Bucharest, 49$54,1984$.

[2] I. Ekeland. On the variational principle. J. Math. Anal. Appl, 47(2):324-353, 1974.

[3] R. S. Hamilton. The inverse function theorem of Nash and Moser. Bull. Amer. Math. Soc, 7(1):65-222, 1982.

[4] H. Hogbe-Nlend. Bornologies and functional analysis, volume 62 of North-Holand Mathematical Studies. 1977.

[5] H. Keller. Differential calculus in locally convex spaces, volume 417 of Lecture Notes in Mathematics. Berlin: Springer-Verlag, 1974.

[6] J. Mawhin, M. Willem. Origin and evolution of the Palais-Smale condition in critical point theory. J. Fixed Point Theory App., 2(7):265-290, 2010.

[7] K-H. Neeb. Toward a Lie theory of locally convex groups. Japan. J. Math., 1(2):291468, 2006.

[8] R. S. Palais, S. Smale. A generalized Morse theory. Bull. Amer. Math. Soc., 70(1):165172, 1964.

[9] J. H. Qiu. Ekeland's variational principle in Fréchet spaces and the density of extremal points. Studia Math., 168(1):81-94, 2005. 
A generalized Palais-Smale condition in the Fréchet space setting

[10] T. Wurzacher. Fermionic second quantization and the geometry of the restricted Grassmannian. Infinite dimensional Kähler manifolds (Oberwolfach, 1995), DMV Sem., 31:287-375, 2001.

Received: December 21, 2017, accepted: February 27, 2018.

Kaveh Eftekharinasab

Topology lab., Institute of Mathematics of NAS of Ukraine, Tereshchenkivska ST. 3, KYIV, 01601 Ukraine

Email: kaveh@imath.kiev.ua 